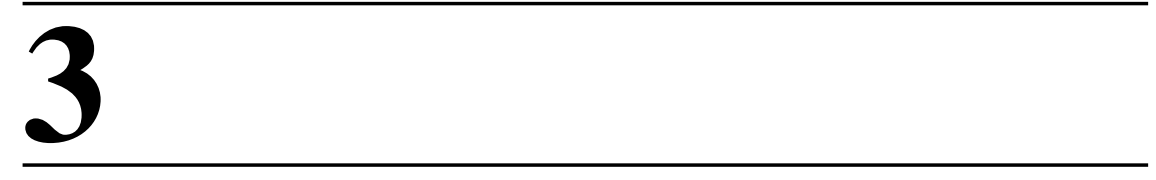

\title{
NANOMATERIALS FOR NEURAL REGENERATION
}

\author{
Melike Sever, Busra Mammadov, Mevhibe Gecer, \\ Mustafa O. Guler, and Ayse B. Tekinay \\ Institute of Materials Science and Nanotechnology, National Nanotechnology \\ Research Center (UNAM), Bilkent University, Ankara, Turkey
}

\subsection{INTRODUCTION}

\subsubsection{Extracellular Matrix of Central Nervous System}

The central nervous system (CNS) consists of a dense network of cells leaving a smaller volume for the extracellular matrix (ECM) components (10-20\% for the brain unlike most other tissues (Cragg, 1979)). Before 1971, CNS was thought to be totally free of ECM and consisting just of a very dense network of cells (Tani and Ametani, 1971). Yet, this small amount is sufficient to modulate the survival, neurite outgrowth, and synaptic plasticity of neurons. Given its importance in modulation of cell function, neural ECM undergoes prominent changes during maturation of the nervous system. Embryonic and postnatal ECM is rather a loose network, while there occurs some condensation throughout adulthood starting from the postnatal period, according to specific needs of neural cells (Bandtlow and Zimmermann, 2000; Dityatev and Schachner, 2003). In fact, the balance among ECM components is so important that any imbalance leads to massive degeneration in the CNS.

Therapeutic Nanomaterials, First Edition. Edited by Mustafa O. Guler and Ayse B. Tekinay.

(C) 2016 John Wiley \& Sons, Inc. Published 2016 by John Wiley \& Sons, Inc. 
Being quite different from that of other tissues, ECM content also differs dramatically between specific locations in the CNS. Two different ECM types are deposited in the basement membrane, perineuronal nets (PNN) and gap filling interstitial matrix. Basement membrane (basal lamina) is the one that is more similar to ECM of other tissues in terms of its components. Collagen, entactin (laminin (LN)-nidogen complexes), fibronectin (FN), dystroglycan, and perlecan are the predominant proteins and proteoglycans (PGs) of basement membrane (Lau et al., 2013). This structure is associated with blood vessels and is important in the stabilization of the blood-brain barrier (Baeten and Akassoglou, 2011).

PNN are the dense network of matrix around cell bodies that is mainly responsible for maintenance of synaptic plasticity. Main components of PNN are hyaluronan, chondroitin sulfate proteoglycans (CSPGs), and tenascins (Bonneh-Barkay and Wiley, 2009; Lau et al., 2013). Being absent in the embryonic tissue, these structures form a barrier around the cells preventing the formation of new synaptic connections while preserving the ones already formed. Mammalian nervous system has a comparable plasticity that continues through postnatal development until maturation of the CNS. During this period, named as critical period, the nervous system continues to develop along with making new connections influenced by experience. Plasticity declines upon closure of the critical period, the time of which coincides with the formation of PNN indicating them as barriers to formation of new connections (Bonneh-Barkay and Wiley, 2009).

Being absent from PNN, small amounts of fiber-forming collagens and elastins along with FN and LN are present in the interstitial matrix. Sulfated PGs and hyaluronan along with tenascins are the main components of this ECM (Lau et al., 2013).

PGs are the predominant components of CNS ECM. PGs are classified into four main groups depending on the GAG chain: CSPGs, heparan sulfate proteoglycans (HSPGs), dermatan sulfate proteoglycans (DSPGs), and keratan sulfate proteoglycans (KSPGs). CSPGs and HSPGs are the main PGs of the CNS, CSPGs being the most abundant (Bonneh-Barkay and Wiley, 2009). Each group is also composed of a variety of PGs due to different protein cores carrying GAG chains. Lecticans are the most abundant CSPG type in the brain, which are classified into four subgroups. Neurocan and brevican are neural tissue-specific lecticans, while aggrecan and versican are common to ECM of other tissues (Yamaguchi, 2000). Besides sulfated GAGs attached to protein cores, hyaluronic acid (HA) is also abundant in the CNS tissues. HA is a large polymeric carbohydrate chain that is nonsulfated and does not contain a protein core. It binds to other PGs and forms a dense mesh-like ECM structure in PNN (Kwok et al., 2010). 
Depending on the protein core and GAG chain, PGs in the CNS are permissive or nonpermissive to neurite extension. GAGs can exert their effects on cells either by inducing direct signaling through cell surface receptors (like HA achieves through CD44 receptor) or by their affinity to growth-active molecules. Affinity of HSPGs to growth factors is quite important in the modulation of neuronal function, especially through FGF signaling (improved upon syndecan binding to FGF (Walicke, 1988)). CSPGs can also bind to a range of molecules including semaphorin 5A, binding to which is found to change permissive nature of semaphorin to inhibitory (Kantor et al., 2004). Binding of CSPGs to other ECM proteins, such as tenascin and LN, blocks axon growth-promoting ability of these proteins and thus inhibits axon growth (Mckeon et al., 1995). While binding of CSPG to LN is inhibitory, binding by HSPG improves axonal elongation (Riopelle and Dow, 1990). CSPGs can also inhibit neural regeneration by limiting calcium availability by binding either to extracellular calcium or to calcium channels, thereby preventing the uptake by neurons (Sharma et al., 2012). Binding of CSPGs to PTPsigma receptor on neurons leads to phosphorylation of neurotrophin receptors TrkB and TrkC, possibly leading to a downregulation of responsiveness to neurotrophins (Faux et al., 2007). Binding of HSPGs to the same receptor in a competitive manner (as in the case of semaphorin 5A) abolishes CSPG inhibitory effect on neurotrophin responsiveness (Kantor et al., 2004). Inhibitory effects of CSPGs are either due to the protein core or sulfated GAG chain, depending on the CSPG type. For versican, neurocan, and phosphacan, protein core is indispensable in axonal inhibition, while GAG chain is responsible for inhibition by aggrecan and brevican. Chondroitinase treatment, which degrades the GAG chain, eliminates the inhibitory effect of aggrecan and brevican but not of the other CSPGs (Maeda and Noda, 1996; Margolis et al., 1996; Niederost et al., 1999; Schmalfeldt et al., 2000; Snow et al., 1990; Yamada et al., 1997).

Although limiting the regenerative capacity of CNS with their inherent inhibitory properties, CSPGs have indispensable role in neural development, both for cell migration and formation of neural connections. Neural crest cells avoid migration through local environments with CSPG abundance. The presence of CSPGs along their way is a trajectory for them to migrate to their defined positions (Kubota et al., 1999). Repulsive nature of CSPGs for growth cones is also quite important in axonal pathfinding and correct formation of neural networks during embryonic development as well as postnatal development in critical period. Treating developing nervous system with chondroitinase ABC leads to various axon pathfinding errors, verifying the importance of 
CSPGs in the process (Chung et al., 2000). CSPGs are also important in adult CNS for proper stabilization of synaptic connections and prevention of improper axonal sprouting and formation of disorganized neural networks (Carulli et al., 2006).

Having a regulatory function on neural network formation during development and keeping connections made stable, CSPGs are quite important in healthy CNS. However, they should be kept in balance in order not to overinhibit neural functions. This balance becomes disorganized upon a trauma leading to production of reactive astrocytes. Astrocytes start to proliferate and deposit dense ECM in the injury site and fill the gap caused by the injury. This preserves the overall structure of the tissue, stabilizes blood-brain barrier, and limits the damage to injury site, thereby preventing secondary damage (Faulkner et al., 2004). Although being important for the overall health of the CNS, the ECM that is deposited hastily by astrocytes is responsible for the failure of neuronal regeneration. This de novo produced tissue is called "glial scar," and it is impermissive to any axonal sprouting due to the abundance of CSPGs. Hence, nearby cells that are healthy cannot enter into the area and thus cannot make new synaptic connections to tolerate the loss of the ones in the injury site. Furthermore, synaptic connections by surviving cells in the injury site cannot be made either due to the inhibitory effect on plasticity.

Besides CSPGs, myelin components are also inhibitory for neural regeneration after CNS injury. The major inhibitory myelin components can be listed as myelin-associated glycoprotein (MAG), Nogo-A, oligodendrocyte myelin glycoprotein $(\mathrm{OMgP})$, and myelin lipid sulfatide (Winzeler et al., 2011; Yiu and He, 2006). Myelin inhibitors MAG, Nogo-A, and OMgP are found to exert their inhibitory effect through binding to Nogo-66 receptor 1 (NgR1) on neurons (Domeniconi et al., 2002; Fournier et al., 2001; Liu et al., 2002; Wang et al., 2002). The presence of NgR1 is essential for growth cone collapse upon encountering myelin inhibitors (Chivatakarn et al., 2007; Kim et al., 2004). Paired immunoglobulin-like receptor B (PirB) and its human homolog (LILRB2) are recently found as another receptor for these three myelin inhibitors, and blocking this receptor along with the NgR1 largely eliminates myelin inhibition of neurite outgrowth (Atwal et al., 2008).

The third type of CNS inhibitory molecules includes axon guidance molecules semaphorin and ephrin. Having a role in network stabilization by limiting neurite outgrowth, most semaphorins are inhibitory for neurons. In the glial scar, class 3 semaphorins (Sema3s) contribute to the inhibitory environment in a CSPG-dependent manner. Interfering CSPG-Sema3 interaction eliminates the inhibitory nature of this molecule in vitro (Pasterkamp and Verhaagen, 2006). Besides Sema3s, membrane-associated semaphorins 
like Sema4D are also upregulated in the glial scar and inhibit neurite outgrowth (Moreau-Fauvarque et al., 2003). Ephrins exert their inhibitory effect by binding to EphA and EphB receptor tyrosine kinase on neural cell surface. EphrinB3 is expressed in CNS myelin, ephrin B2 is upregulated upon injury by reactive astrocytes, and ephrinA5 expression is found to be increased in cortical lesion, and they all inhibit neurite outgrowth (Benson et al., 2005; Bundesen et al., 2003; Carmichael et al., 2005).

\subsubsection{ECM of Peripheral Nervous System}

The reaction of the nervous tissue to any injury leading to scar tissue formation acts as a barrier for regeneration in the CNS, while it supports regeneration in the peripheral nervous system (PNS). Different types of glial cells in the CNS and the PNS, astrocytes and Schwann cells, lead to the formation of completely different environments for axons struggling to heal from an injury. While astrocytes produce a growth-inhibitory environment consisting of chemorepulsive guidance molecules, Schwann cells produce a growth promoting environment by secretion of supportive ECM molecules like neurotrophins as well as dealing with the clearance of inhibitory myelin products after injury. A chain of events occurring after an injury in the peripheral nerve leads to the formation of a permissive environment for axonal regeneration. Wallerian degeneration, occurring in the distal stump of the injured nerve, leads to fragmentation of the damaged axon and associated myelin products. Fragmented products are then cleared by macrophages that are recruited to the injury site (Chen et al., 2007). Myelin-associated proteins are known to inhibit axonal elongation, and their clearance after injury in the PNS forms a permissive environment as opposed to that in the CNS. While the distal stump undergoes Wallerian degeneration, axonal regeneration starts from the proximal end. Schwann cells dedifferentiate and start to proliferate. They myelinate newly elongating axon, thus providing an electrical insulation to the regenerating axon (Fawcett and Keynes, 1990).

Permissive environment, axon guidance cues, and intrinsic growth capacity of peripheral nerves are crucial in successful regeneration of the PNS. Unlike their CNS counterpart, peripheral nerves have an intrinsic growth capacity that is activated upon injury. Cyclic adenosine monophosphate (cAMP) level is upregulated upon peripheral nerve injury, and it activates protein kinase A (Neumann et al., 2002; Qiu et al., 2002). Protein kinase $\mathrm{A}$ is thought to take role in cytoskeletal reorganization by inhibiting RhoA. RhoA is normally activated by the presence of myelin debris, and its activation leads to halting of cytoskeletal reorganization required for axonal 
elongation. Hence, its inhibition by protein kinase A makes axon's cytoskeletal dynamics favorable for regeneration (Snider et al., 2002). Besides, cAMP has a transcriptional role by activating cAMP response element binding protein (CREB), which in turn enables cell to overcome myelinassociated inhibition of neurite outgrowth (Gao et al., 2004). Upregulation of intracellular cAMP levels leads to an increase in cell surface localization of TrkB receptors also, which in turn increases axons' responsiveness to neurotrophins (Liu et al., 2011; Meyer-Franke et al., 1998).

Structurally different from that of the CNS, the PNS is abundant in basal lamina. Secreted by Schwann cells, basal lamina of the peripheral nerve tissue contains LN, type IV collagen, and HSPGs (Bunge et al., 1990). Being present in healthy peripheral nerve, basal lamina production is upregulated in the injured nerve (Chen et al., 2007). Besides its importance in myelination of nerves by Schwann cells, basal lamina components are also important for axonal elongation. $\mathrm{LN}$ is a positive regulator of neurite outgrowth, and it is overexpressed after peripheral nerve injury. Abolishing the expression of LN by Schwann cells impairs axon regeneration severely. Besides their role in neurite outgrowth, LN are also important for Schwann cells' proper differentiation and myelination of growing axons (Chen and Strickland, 2003). In addition to LN, NGF, BDNF, GDNF, IGF, CNTF, and FGF-2 are also upregulated in the injured peripheral nerve. Increased amount of these growth factors in the injury site provides better axonal elongation along with proper myelination by Schwann cells (Giger et al., 2010).

Myelin inhibition, an important problem for CNS regeneration, is not a big deal in the PNS. There are several routes by which peripheral nerves deal with this problem. Responsiveness of the axon to myelin products is thought to be different in the PNS when compared to the CNS, which is actually related to the intrinsic growth capacity of peripheral nerves. Intracellular cAMP levels, for example, determine whether MAG will act as a growth inhibitory molecule or not. Besides, lack of some myelin proteins in PNS myelin is thought to make PNS myelin less inhibitory through other major components such as MAG that it still consists (Chen et al., 2007). Clearance of myelin from the injury site is another important event, which does not take place in the CNS. It is rapidly cleared away from the injured peripheral nerve by hand-to-hand functioning of Schwann cells and macrophages. In the early stages after injury, Schwann cells are the main phagocytic cells in the injured nerve, and they start myelin clearance as early as $5 \mathrm{~h}$ after injury. They secrete phospholipase A2 that degrades phosphatidylcholine, which is abundant in myelin. Lysophosphatidylcholine is produced by hydrolysis of phosphatidylcholine, and it has a myelinolytic activity (Martini et al., 2008). Schwann cells also recruit macrophages to the 
injured peripheral nerve by secreting several chemokines including TNF-a, LIF, Il-1a, Il-1b, and Il-6 (Gaudet et al., 2011). Infiltration of macrophages to the nerve is essential in clearing myelin debris from the distal stump that undergoes Wallerian degeneration. Inhibitory myelin proteins are not cleared in the CNS due to the lack of macrophages in the lesion site, the infiltration of which is restricted by the blood-brain barrier.

Upon peripheral nerve injury, fibrinogen from the blood starts to infiltrate into the nerve where it is converted to fibrin. Accumulated fibrin prevents Schwann cell migration to the injury site and prevents remyelination (Akassoglou et al., 2002). Schwann cells upregulate production of tissue plasminogen activator (tPA) and activates fibrinolytic pathway thereby. Lysis of fibrin enables Schwann cell to enter the injured nerve and start myelination of the growing axon (Akassoglou et al., 2000).

\subsubsection{Urgent Need for Materials to Induce Regeneration in Nervous Tissue}

Due to the inherent lack of regeneration in the CNS, brain and spinal cord trauma makes quite a high number of people suffer from the lifelong effects. Ineffective therapies cause almost all survivors to continue their life with the aid of others.

Although the PNS is better than the CNS in terms of its regenerative potential, injuries leading to injuries to long nerve segments lead to disappointing therapeutic results. End-to-end bridging is not possible due to the tension that will lead to secondary injury, and harvesting long segments of the nerve from other sides of the body for autografting is also out of question. Commercially available nerve tubes, although providing some axonal elongation, are not always effective.

These clinical challenges make development of new therapies for nervous system injuries and disorders crucial. There are plenty of papers published in the area showing the efforts to meet this requirement. However, to be successful, one should understand the native tissue properties and requirements of the resident cells. For example, neural cells are electrically active cells, and conductive materials might support regeneration better. Besides, incorporating biological signals such as short peptides that bind to cell surface receptors and induce axonal elongation is found to lead promising results (Mammadov et al., 2012a). Biocompatibility of the material to be used is also essential, and long-term toxicity assessments should be carried out before planning a clinical use. Biodegradable materials are advantageous, if no lifelong therapy is desired. However, end products after degradation should also be nontoxic to avoid any harm to regenerating tissue de novo. 


\subsection{NANOMATERIALS FOR NEURAL REGENERATION}

\subsubsection{Physical Functionalization of Nanomaterials to Induce Neural Differentiation}

During the development of the nervous system, ECM plays an important role to support neuronal maturation and neurite extension. Cell adhesion, proliferation, and differentiation are other important significant criteria to be considered when designing a nanomaterial for neural tissue engineering. The main goal of regenerative medicine is to enhance tissue regeneration and healing after injury or disease leading to degeneration of the tissue of interest. Guidance of cell behavior by the features of a material at cell-biomaterial interfaces has significant importance, and developing novel biomaterials with certain surface modifications to induce controlled cell function would be beneficial for improving the therapeutic potential of current regenerative medicine protocols. The biodegradability and biocompatibility are the most important features of synthetic biomaterials. Physical properties of a material should also be taken into account in addition to biological and chemical properties when designing biomaterials for tissue engineering applications. Physical properties including stiffness, dimensionality, substrate topography, and electrical conductivity are important parameters for scaffold functionalization to induce neural differentiation (Fig. 3.1).

\subsubsection{Effects of Mechanical Stiffness on Cellular Behavior}

Mechanical properties of the microenvironment are important regulators of cell properties including morphology (Bard and Hay, 1975) and motility (Halliday and Tomasek, 1995) of the cells as well as differentiation (Engler et al., 2006). When designing a scaffold for neural differentiation, the mechanical properties of the scaffold should be similar to that of brain tissue, which is below $1 \mathrm{kPa}$ (Georges et al., 2006).

In mechanically tunable matrices and substrates, it was shown that PNS cells (Flanagan et al., 2002; Willits and Skornia, 2004) and PC12 cells (Gunn et al., 2005; Leach et al., 2007) displayed variable neurite outgrowth and branching according to the substrate stiffness. In the case of neural stem cells (NSCs), the influence of the mechanical properties of the substrate on cell behavior has been studied (Saha et al., 2008). According to this study, NSCs prefer to differentiate into neurons with intermediate substrate stiffness $(500 \mathrm{~Pa})$, while increased stiffness favors the differentiation into astrocyte lineage (Saha et al., 2008). Also, it was shown that while substrate 


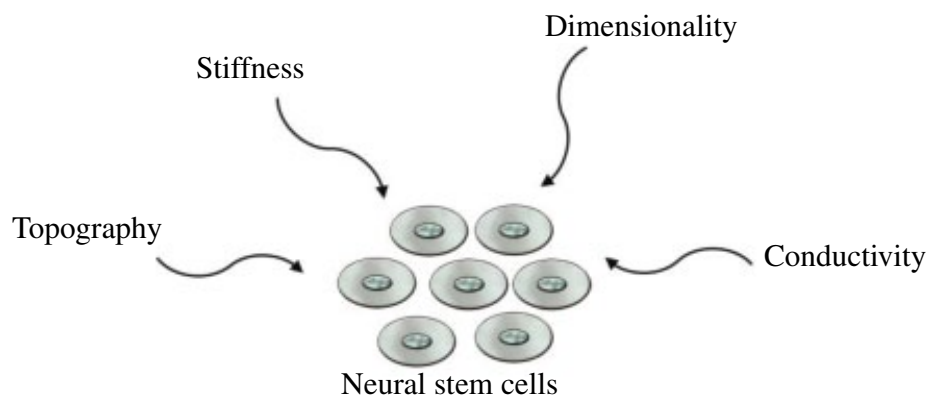

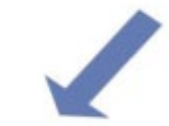

Neuron

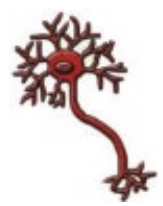

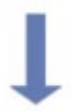

Astrocyte

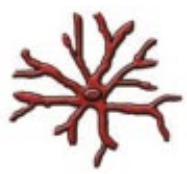

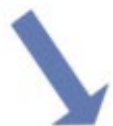

Oligodendrocyte

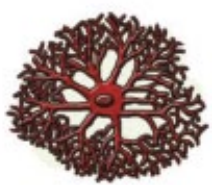

FIGURE 3.1 Physical properties to induce neural differentiation of neural stem cells (NSCs).

stiffness did not affect the number of neurons differentiated from NSCs, soft substrates promote neuronal maturity, and neurons differentiated on soft substrates displayed long neurites in the absence of added growth factors. In contrast, neurons showed a bipolar immature morphology on stiff substrates (Teixeira et al., 2009).

LN-coated methacrylamide chitosan (MAC) hydrogels with different stiffnesses were used to understand the contribution of substrate stiffness to neural stem/progenitor cell (NSPC) differentiation and proliferation. It was demonstrated that an optimal stiffness is present for both proliferation $(3.5 \mathrm{kPa})$ and differentiation of NSPCs to neurons $(<1 \mathrm{kPa})$ (Leipzig and Shoichet, 2009).

The effect of stiffness was also studied on human mesenchymal stem cells (hMSCs). This effect was investigated by using three-dimensional (3D) porous scaffolds generated by type I collagen (Col) and HA. Using different concentrations of 1-ethyl-3-(3-dimethylaminopropyl)carbodiimide (EDC) as a crosslinking agent, the elastic modulus of the 3D substrates was modified, and the stiffness was controlled in the range of 1-10 kPa. Results showed that mesenchymal stem cells (MSCs) were likely to differentiate into neurons with $1 \mathrm{kPa}$ while they preferred glial 
lineage in the scaffold with $10 \mathrm{kPa}$ stiffness verified with experiments focused on upregulation of neuronal mid and late protein markers after longer mechanical induction time for 21 days (Her et al., 2013).

In another study, spinal cord neurons were used to study cellular responses to substrate compliances. DNA crosslinked hydrogels with stiffness of approximately $100 \mathrm{~Pa}$ to $30 \mathrm{kPa}$ were produced. DNA gel stiffnesses were modulated by changing the length of crosslinker, monomer concentration, and level of crosslinking. Although this variation in stiffness did not significantly affect the primary dendrite length, it was found that stiffer gels caused extending more primary dendrites and shorter axons with the reduction in focal adhesion kinase (FAK), suggesting the involvement of FAK in neuronal mechanosensing and neuritogenesis in response to stiffness. It provides cues for the design of scaffolds for neural tissue engineering depending on the mechanical properties (Jiang et al., 2008).

\subsubsection{Effects of Dimensionality on Cellular Behavior}

In vitro models are important tools to study cell behavior and fate in a highly controlled manner. Although two-dimensional (2D) cell cultures are commonly used in differentiation studies, 3D cell cultures are important in vitro models to fill the gap between $2 \mathrm{D}$ cell culture experiments and in vivo studies. Thus, use of 3D models is important to the study regeneration of neural tissue, which has a very low regeneration capacity. 3D models better mimic the ECM of neural cells in terms of providing support and enhanced diffusion of oxygen and nutrients.

There are fundamental differences between cells grown on a monolayer surface and in a 3D culture. Cells grown in 3D cultures have been shown to display longer neurite outgrowth and higher levels of survival as well as different patterns of differentiation when compared to 2D monolayers (Bellamkonda et al., 1995; Pardo and Honegger, 2000). Also, hippocampal neurons cultured on a 3D aragonite matrix displayed higher survival as compared to 2D counterparts with higher-density network formation (Peretz et al., 2007). In another study, 3D cell culture system with the functional motifs Arg-Gly-Asp (RGD), bone marrow homing peptide 1 (BMHP1), and BMHP2 was developed for the culture of adult NSCs. The proliferation and differentiation of NSCs were supported by these scaffolds through allowing a satisfactory supply of nutrients and oxygen (Cunha et al., 2011).

Stupp and coworkers generated a 3D network of nanofibers formed by self-assembly of peptide amphiphile (PA) molecules to encapsulate neural progenitor cells. They used neurite-promoting LN epitope IKVAV, and this artificial nanofiber system gave rise to rapid differentiation of neural 
progenitor cells into neurons while suppressing the astrocyte differentiation (Silva et al., 2004).

Also, LN-derived IKVAV-linked self-assembling peptide RADA16 was used to form a 3D matrix as a functional peptide-based scaffold. NSCs were encapsulated within this 3D peptide hydrogel to increase the reconstruction of the injured brain. The signal coming from the extended IKVAV sequence directs the adhesion of NSCs and leads to neuronal differentiation. In addition to in vitro studies, rat brain surgery model was generated to illustrate the damage in cerebral neocortex/neopallium loss. Injection of NSC-encapsulated 3D hydrogel filled the cavity caused by injury. They revealed enhanced survival of encapsulated NSCs and reduced glial scar formation with histological analysis. This 3D hydrogel model provided improvement in brain tissue regeneration 6 weeks after posttransplantation (Cheng et al., 2013).

\subsubsection{Effects of Substrate Topography on Cell Behavior}

The natural environment of the cells has complex topographical cues, which are different between different surfaces used for in vitro cultures. Nanotopography is an important tool to guide cell differentiation, and it can be modified in size and shape according to desired application. Interaction of the cells with nanotopographical features can induce different responses including changes in cell morphology (Dalby et al., 2002a), adhesion (Gallagher et al., 2002), proliferation (Dalby et al., 2002b), and gene regulation (Dalby et al., 2002c). It is important to understand that neurite growth along topographical patterns is important for tissue engineering applications in neurology.

Cecchini et al. analyzed time-lapse images to understand the differentiation dynamics of PC12 cells on nanopatterned biocompatible substrates. Depth gratings were produced on tissue-culture polystyrene substrates by nanoimprint lithography, and the results suggested that the substrate topography can act cooperatively with nerve growth factor (NGF) signaling to regulate differentiation and neurite outgrowth (Cecchini et al., 2007).

Embryonic stem cell (ESC) differentiation into specific cell lineages is one of the major concerns in regenerative medicine. In order to analyze the effect of different nanostructures on differentiation of ESC-derived neuronal precursors into neuronal lineage without biochemical factors, patterns in polydimethylsiloxane (PDMS) consisting of groove and pillar arrays of submicrometric lateral resolution were produced. It was found that neuronal differentiation was enhanced when neuronal precursors were cultured on nanopillars compared to flat PDMS substrates and neurites grew mostly on the top of the higher pillars (Migliorini et al., 2011). 
Polyurethane acrylate nanoscale ridge/groove pattern arrays were generated to induce the differentiation of hESCs into a neuronal lineage without the use of any differentiation-inducing agents. The hESCs were found to differentiate into various neurons, but not into glial cells, such as astrocytes, as evidenced by immunofluorescence results (Lee et al., 2010).

In addition, nanotopographical control of neuronal polarity was studied, and the interaction between focal adhesions and topographical features was exploited to design a set of scaffolds made of cyclic olefin copolymer (COC), yielding control over neuronal polarity establishment and neurite pathfinding. By this way, specific neuronal polarity states were selectively favored by a set of biocompatible textured scaffolds, and it was demonstrated that this selection can be tailored through varying the topographical constraint applied to the maturation of focal adhesions during neuritogenesis (Ferrari et al., 2011).

\subsubsection{Effects of Electrical Conductivity on Cell Behavior}

Neurons are electrically excitable cells, and as a result of their inherent nature, they are capable of transmitting electrochemical signals throughout the nervous system, making them highly sensitive to electrical stimuli. The action potential generated at the synapse is the key component of neural communication, so electrical conductivity is an important physical property to enhance neural cell activity (Hodgkin and Huxley, 1952). Providing electrical conductivity to the scaffolds and electrical stimulation of the cells cultured on these scaffolds might be useful to improve synaptic connections.

One of the new aspects of scaffold generation is the design of conductive substrates, which provide external electrical stimuli to the stem cells, because these stimuli can affect the cell behavior including proliferation, migration, and differentiation (Chang et al., 2011; Huang et al., 2012).

Electrical conductivity can be provided to the scaffold through utilization of conductive polymers and carbon-based materials, which include carbon nanotubes (CNTs), graphite, and graphene (Kotov et al., 2009). Combining the conductivity of CNT with the alignment of poly(L-lactic acid) (PLLA) nanofibers supports and increases the neural differentiation of mouse embryonic stem cells (mESCs), which was detected by enhanced expression of mature neuronal markers such as Map-2 and NSE even in the absence of direct electrical stimulation (Kabiri et al., 2012).

The effect of electroactive fibers on responses of neuronal cells was also investigated (Bhang et al., 2012). In this study, electrically active electrospun 
fibers were generated from blends of poly(lactide-co- $\varepsilon$-caprolactone) and a conductive polymer, polyaniline (PAni). These electrically conductive fibers sustained stable electrical properties in cell culture conditions for up to 7 days and enhanced NGF-induced neurite outgrowth of PC12 cells verified with morphological changes and the expression of differentiation proteins.

\subsection{CHEMICAL AND BIOLOGICAL FUNCTIONALIZATION OF NANOMATERIALS FOR NEURAL DIFFERENTIATION}

By mimicking several unique characteristics of the natural environment of cells, synthetic materials for neural regeneration can be improved chemically and biologically. Especially bioactivation of materials can be achieved by addition of small chemical moieties to the scaffold particularly found in specific tissues or addition of biologically active molecules derived from natural ECM.

\subsubsection{Effects of Biologically Active Molecules on Cell Behavior}

Incorporation of the bioactive regions of ECM proteins that are used to direct and maintain the cell differentiation can be used to modify or reconstruct the scaffolds to be used as cellular environments (Ma et al., 2008). With the modification of these bioactive proteins, newly designed materials may be developed to enhance differentiation efficiency.

In order to control differentiation of NSCs for clinical applications, construction of new composite biomaterials to find an ideal combination of ECM proteins and growth factors is another approach. To induce NSC differentiation into specific cell types, gold surfaces modified by the photoassisted patterning of an alkanethiol self-assembled monolayer (SAM) were used for immobilization of natural and synthetic matrices (FN, LN, poly(ethyleneimine) (PEI), PLL, RGD, IKVAV) along with growth factors such as epidermal growth factor (EGF), ciliary neurotrophic factor (CNTF), NGF, and neurotrophin-3 (NT-3). This study showed that immobilized LN/NGF, FN/NGF, Pro-F/NGF, FN/NT-3, and Pro-F/NT-3 on spots promoted more neuronal differentiation, while astroglial differentiation was promoted with FN/CNTF and Pro-F/CNTF among other combinations (Nakajima et al., 2007).

The fate of NSCs depends on the ECM-cell interactions, especially integrin interactions, which are directly connected with laminin proteins (Nakajima et al., 2007). In order to evaluate the impact of the laminin microenvironment on primary murine neurosphere culture, methylcellulose 
(MC) scaffolds functionalized with laminin-1 (MC-x-LN1) were used as a scaffold. This study revealed that MC-x-LN1 induces NSC survival and maturation compared to that of MC only controls. Moreover, higher neuronal and oligodendrocytic differentiation was observed in MC-x-LN1 scaffold than in MC controls. Correspondingly, LN1 production and $\alpha_{6} \beta_{1}$ integrin levels were significantly extended on MC-x-LN1 than in MC controls (Stabenfeldt et al., 2010).

Aerogels were also used as a class of material, with tunable properties including chemical, physical, and surface properties. In order to evaluate the ability of polyurea-crosslinked silica aerogels (PCSA) coated with PLL (a synthetic polymer of L-lysine), basement membrane extract (BME) or LN1 was used for culture of DRG neurons. PCSA displayed the ability to induce directional differentiation and growth of DRGs. Furthermore, laminin was demonstrated to be a highly efficient surface material for attachment and growth of dorsal root ganglion (DRG) neurons on PCSA (Sabri et al., 2012).

Functional polymer scaffolds produced by covalent modification of PLLA nanofibers with laminin were also used to mimic bioactive properties of the ECM proteins by electrospinning technique. Cultured PC12 cells differentiated into neural cells in the presence of NGF more efficiently on these modified surfaces than those on the unblended PLLA. Using this modification technique, different types of biocompatible polymers with a significant potential in nerve tissue regeneration applications can be acquired (Koh et al., 2008). In another study, fibers made up of poly(l-lactic acid)co-poly-(3-caprolactone) and collagen were fabricated by electrospinning to investigate human bone marrow-derived MSCs for neural differentiation on this nanofibrous scaffold. Neural induction of MSCs was carried out by culturing in the presence of $\beta$-mercaptoethanol, EGF, NGF, and BDNF. These results showed that the differentiation of MSCs into neurons was comparatively higher on Poly(1-lactic acid)-co-poly-(3-caprolactone)collagen surfaces than those on Poly(l-lactic acid)-co-poly-(3-caprolactone) surface without collagen treatment. Moreover, the expression of neuronal markers neurofilament (NF) and nestin were observed within multipolar elongations by immunofluorescence (Prabhakaran et al., 2009).

\subsubsection{Effects of Chemical Groups on Cellular Behavior}

Chemical modification by specific terminal groups can modulate NSCs' response to different scaffolds including their differentiation. In the NSC extracellular environment, high abundance of specific chemical moieties is believed to direct neural differentiation, so substrates functionalized with chemical groups may be beneficial in controlling the behavior of NSCs. 
Glass surfaces chemically modified with hydroxyl (-OH), methyl $\left(-\mathrm{CH}_{3}\right)$, amino $\left(-\mathrm{NH}_{2}\right)$, carboxylate $(-\mathrm{COOH})$, mercapto $(-\mathrm{SH})$, and sulfonate $\left(-\mathrm{SO}_{3} \mathrm{H}\right)$ groups were used for culture of NSCs to investigate the commitment of stem cells to a specific neural subtype during differentiation. $-\mathrm{SO}_{3} \mathrm{H}$-modified surfaces were found to induce oligodendrocyte differentiation more. $-\mathrm{NH}_{2}$ had a tendency to induce more neuronal differentiation and inhibit the oligodendrocyte differentiation of NSCs in comparison with $-\mathrm{COOH}$ (Ren et al., 2009).

Biomimetic polymeric scaffolds have recently become the focus point of research on nerve regeneration. Another method of chemical modification of polymeric scaffolds is synthesizing critical protein, acetylcholine, a neurotransmitter, and a neuromodulator in both the PNS and the CNS. In the PNS, acetylcholine binds to acetylcholine receptors on skeletal muscle fibers, and it opens the ligand-gated sodium channels. In the CNS, damages in the cholinergic system have been shown to be associated with memory deficit in diseases such as Alzheimer's disease. Acetylcholinelike biomimetic polymers including a bioactive unit mimicking the acetylcholine, a bioinert unit poly(ethylene glycol) monomethyl ether-glycidyl methacrylate (MePEG-GMA), and dimethylaminoethyl methacrylate (DMAEMA) were synthesized via click chemistry and free radical polymerization technique. Rat hippocampal neurons were used to observe the effect of synthesized polymer that has a significant effect on neuronal growth when 1:60 (mol/mol) ratio of MePEG-GMA to DMAEMA is used (Tu et al., 2011).

Other studies demonstrated that a substrate energy of adhesion (Murnane et al., 2002) generated by chemical heterogeneity, especially in the form of local gradients (Lamour et al., 2009), can be important for the guidance of neurite and neural regeneration on biosynthetic materials. In one study, SAMs of alkylsiloxanes, which had an extensive effect on generating surface energy distribution on glass, were used as a substrate. It was showed that chemical heterogeneity had a crucial impact on PC12 cell differentiation and adhesion within $48 \mathrm{~h}$ without NGF treatment (Lamour et al., 2010).

One of the promising techniques for nerve repair and regeneration is to support and induce axonal growth by application of nanometer-scale materials, particularly nanofibers and nanotubes mimicking the natural tubular structures such as microtubules, ion channels, and axons. Nanotubes can be fabricated from a variety of different materials such as carbon, silicon, synthetic polymers, DNA, lipids, and proteins. CNTs are novel materials that are able to regulate nerve cell behavior. They are composed of sheets of graphite rolled in a continuous cylinder and classified 
as single-walled CNTs (SWCNTs) or multiwalled CNTs (MWCNTs) depending on the number of layers formed (Harrison and Atala, 2007). CNTs can be modified with positively or negatively charged chemical groups such as $-\mathrm{NH}_{2},-\mathrm{COOH}$, and $-\mathrm{SH}$. The positively charged moieties, especially amine groups, may induce neural differentiation by electrostatic interaction with the negatively charged molecules localized on neural cell surface (Hu et al., 2004). PEG is also used to modify SWCNTs and found to reduce the toxicity of CNTs and increase the length of neurites extended by PC12 cells (Malarkey et al., 2008). However, the clinical use of CNTs remains controversial regarding the toxicity and the uncertainty of long-term effects for in vivo studies.

\subsubsection{Effects of Biofunctionalization on Cellular Behavior Through ECM-Derived Short Peptides}

The ECM-derived short peptides are promising candidates to be presented as functional domains on the scaffold surface for use in neural regeneration. These are biocompatible and biodegradable synthetic materials that interact with cell surface receptors similarly to native ECM proteins.

One of the most known ECM-derived peptides is IKVAV sequence derived from laminin. This sequence is a neurite-guiding integrin-binding epitope. Superporous P(HEMA-AEMA) hydrogels were constructed radically as a scaffold by copolymerization of 2-hydroxyethyl methacrylate with 2-aminoethyl methacrylate (AEMA) and ethylene dimethacrylate in the presence of ammonium oxalate crystals. Differentiation of rat MSCs was examined on P(HEMA-AEMA) hydrogels modified with lamininderived IKVAV, as well as on PHEMA, P(HEMA-AEMA)-SH, and P(HEMA-AEMA)-TPy hydrogels. $\beta$-III tubulin, NF, and synaptophysin were expressed highly by fetal NSCs cultured on IKVAV-p(HEMA-AEMA) scaffolds (Kubinova et al., 2010).

Polyamide nanofibers are constituted of nanofibrillar surface that can mimic the spatial organization and porosity of the natural ECM. Primary cerebellar granule, cerebral cortical, hippocampal, motor, and DRG neurons were isolated and cultured on polyamide electrospun nanofibers modified with neuroactive tenascin $\mathrm{C}$-derived peptides. These scaffolds were found to enhance neuronal adhesion, neuritogenesis, and neurite extension in vitro compared to unmodified polyamide scaffolds and PLLcoated glass cover slips (Ahmed et al., 2006).

A hydrogel scaffold produced by fiber-templating method and modified with laminin-derived oligopeptides, CDPGYIGSR and CQAASIKVAV, was used in another study to enhance neurite outgrowth and cell adhesion 
of primary neurons. The results of this study indicated that modification of poly(HEMA-co-AEMA) surface with peptides promoted extension of longer neurites when compared to the control P(HEMA-co-AEMA). Besides, there was no statistical difference between the neurite lengths of cells grown on modified hydrogels and positive control scaffold made of PLL/laminin (Yu and Shoichet, 2005).

In order to mimic the unique properties of natural ECM scaffolds, selfassembled peptide nanofiber system is one of the most versatile scaffolds due to their ease of modification for presenting bioactive epitopes found in ECM proteins or other neural differentiation inducer proteins. PA molecules contain an alkyl tail connected to hydrophobic amino acids and a hydrophilic bioactive epitope. In aqueous solutions, nanofibers form by self-assembly of individual molecules through charge neutralization and hydrogen bonding. Laminin epitope, IKVAV, presented on peptide nanofibers was used to promote differentiation of neural progenitor cells to neurons and for inducing neurite extension. These synthetic scaffolds provided selective differentiation into neurons very rapidly, while less astrocyte differentiation was observed (Silva et al., 2004). By using the self-assembly approach, PA nanofibers can be used to present multiple bioactive epitopes in one nanofiber system. Use of laminin-derived IKVAVPAs along with heparan sulfate-mimicking PAs was found to act cooperatively in enhancing neural differentiation of PC12 cells. Moreover, these neural cells were shown to extend neurites even in the presence of inhibitory environment such as CSPGs on peptide nanofiber scaffolds (Mammadov et al., 2012a). Heparin-mimetic nanofibers were found to bind NGF with higher affinity than control peptide nanofibers. Also the results demonstrated that binding of growth factors to the scaffold did not lead to any loss of NGF bioactivity as demonstrated by PC12 differentiation (Mammadov et al., 2012b).

In peripheral nerve regeneration, PA nanofibers can also be used for the delivery of biological molecules such as sonic hedgehog (SHH) protein. In the animal model, SHH signaling was inhibited in the cavernous nerve in rats, and SHH protein was released by PA nanofiber hydrogels. SHH delivery enhanced the regeneration of the cavernous nerve, reduced penile apoptosis, and improved erectile function (Angeloni et al., 2011). In another study, the combination of the epitope-presenting PA molecules with type I collagen by a hybrid matrix approach sustained neuronal survival and control over dendrite and axon growth of Purkinje cells. While collagen matrix provided a desirable mechanical support, lamininderived PA molecules were used to adjust the bioactivity concentration of the matrix (Sur et al., 2012). 


\subsection{CONCLUSION}

The nervous system is the most complex system of the body due to the complex interactions of neurons with each other, which is hard to reestablish when disordered. Besides its complexity, the inherent fragile nature of neurons combined with the strictly inhibitory environment of the CNS makes regeneration even more difficult, if not impossible. Neurons lost due to a trauma or neurodegenerative disorder are not replenished, except very constrained areas where stem cells reside within the brain. Instead, such pathological conditions usually get even worse due to the proliferation of astrocytes and the inhibitory ECM that they deposit. Neurons that survive after the primary injury cannot make new connections in the scar tissue since they are not allowed to elongate axons. Their nerve terminals start to degenerate eventually leading to cell death resulting in secondary injury. Although the environment and the glial cells in the PNS are not inhibitory but supportive for regeneration, regeneration in PNS injuries is not without problems. Injuries that cause damage in long nerve segments are quite problematic. End-to-end suturing is not preferred to avoid the tension that will lead to secondary injury. Autografting is also not possible as harvesting long segments of nerves from other sides of the body is not possible. Commercially available nerve tubes are the only option for such injuries though they don't offer high success in clinical outcome.

Poor regenerative capacity of the nervous system along with the lack of successful therapies puts a pressure on clinicians and scientists to develop new approaches to relieve the burden of neural injuries and neurodegenerative disorders. Mimicking the healthy ECM of the nervous system by means of its mechanical, chemical, and biological properties is essential to offer neurons an environment where axonal regeneration and new synaptic connections can take place. A variety of materials from polymers to natural materials and synthetic self-assembled systems are currently being developed to fill the gap in the area. To find the optimal material for clinical success in neural regeneration, however, one should consider essential requirement of the material being biocompatible as well as it structural and functional properties' similarity to the natural tissue. Current literature on developing and applying such materials to induce or enhance neural regeneration is reviewed in here. To sum up, we can conclude that it is essential to make a smart design considering all the necessities of the tissue to be applied and currently there has been a lot of effort to generate such materials with clinical success due to their high demand. 


\section{REFERENCES}

Ahmed, I., Liu, H.Y., Mamiya, P.C., Ponery, A.S., Babu, A.N., Weik, T., Schindler, M., and Meiners, S. (2006). Three-dimensional nanofibrillar surfaces covalently modified with tenascin-C-derived peptides enhance neuronal growth in vitro. J Biomed Mater Res A 76, 851-860.

Akassoglou, K., Kombrinck, K.W., Degen, J.L., and Strickland, S. (2000). Tissue plasminogen activator-mediated fibrinolysis protects against axonal degeneration and demyelination after sciatic nerve injury. J Cell Biol 149, 1157-1166.

Akassoglou, K., Yu, W.M., Akpinar, P., and Strickland, S. (2002). Fibrin inhibits peripheral nerve remyelination by regulating Schwann cell differentiation. Neuron 33, 861-875.

Angeloni, N.L., Bond, C.W., Tang, Y., Harrington, D.A., Zhang, S., Stupp, S.I., McKenna, K.E., and Podlasek, C.A. (2011). Regeneration of the cavernous nerve by Sonic hedgehog using aligned peptide amphiphile nanofibers. Biomaterials 32, 1091-1101.

Atwal, J.K., Pinkston-Gosse, J., Syken, J., Stawicki, S., Wu, Y., Shatz, C., and Tessier-Lavigne, M. (2008). PirB is a functional receptor for myelin inhibitors of axonal regeneration. Science 322, 967-970.

Baeten, K.M. and Akassoglou, K. (2011). Extracellular matrix and matrix receptors in blood-brain barrier formation and stroke. Dev Neurobiol 71, 1018-1039.

Bandtlow, C.E. and Zimmermann, D.R. (2000). Proteoglycans in the developing brain: new conceptual insights for old proteins. Physiol Rev 80, 1267-1290.

Bard, J.B. and Hay, E.D. (1975). The behavior of fibroblasts from the developing avian cornea. Morphology and movement in situ and in vitro. J Cell Biol 67, 400-418.

Bellamkonda, R., Ranieri, J.P., Bouche, N., and Aebischer, P. (1995). Hydrogelbased three-dimensional matrix for neural cells. J Biomed Mater Res 29, 663-671.

Benson, M.D., Romero, M.I., Lush, M.E., Lu, Q.R., Henkemeyer, M., and Parada, L.F. (2005). Ephrin-B3 is a myelin-based inhibitor of neurite outgrowth. Proc Natl Acad Sci U S A 102, 10694-10699.

Bhang, S.H., Jeong, S.I., Lee, T.J., Jun, I., Lee, Y.B., Kim, B.S., and Shin, H. (2012). Electroactive electrospun polyaniline/poly[(L-lactide)-co-(epsiloncaprolactone)] fibers for control of neural cell function. Macromol Biosci 12, 402-411.

Bonneh-Barkay, D. and Wiley, C.A. (2009). Brain extracellular matrix in neurodegeneration. Brain Pathol 19, 573-585.

Bundesen, L.Q., Scheel, T.A., Bregman, B.S., and Kromer, L.F. (2003). EphrinB2 and EphB2 regulation of astrocyte-meningeal fibroblast interactions in response to spinal cord lesions in adult rats. J Neurosci 23, 7789-7800. 
Bunge, M.B., Clark, M.B., Dean, A.C., Eldridge, C.F., and Bunge, R.P. (1990). Schwann-cell function depends upon axonal signals and basal lamina components. Ann N Y Acad Sci 580, 281-287.

Carmichael, S.T., Archibeque, I., Luke, L., Nolan, T., Momiy, J., and Li, S.L. (2005). Growth-associated gene expression after stroke: evidence for a growthpromoting region in peri-infarct cortex. Exp Neurol 193, 291-311.

Carulli, D., Rhodes, K.E., Brown, D.J., Bonnert, T.P., Pollack, S.J., Oliver, K., Strata, P., and Fawcett, J.W. (2006). Composition of perineuronal nets in the adult rat cerebellum and the cellular origin of their components. J Comp Neurol 494, 559-577.

Cecchini, M., Bumma, G., Serresi, M., and Beltram, F. (2007). PC12 differentiation on biopolymer nanostructures. Nanotechnology 18, 505103.

Chang, K.A., Kim, J.W., Kim, J.A., Lee, S., Kim, S., Suh, W.H., Kim, H.S., Kwon, S., Kim, S.J., and Suh, Y.H. (2011). Biphasic electrical currents stimulation promotes both proliferation and differentiation of fetal neural stem cells. PLoS One 6, e18738.

Chen, Z.L. and Strickland, S. (2003). Laminin gamma 1 is critical for Schwann cell differentiation, axon myelination, and regeneration in the peripheral nerve. J Cell Biol 163, 889-899.

Chen, Z.L., Yu, W.M., and Strickland, S. (2007). Peripheral regeneration. Annu Rev Neurosci 30, 209-233.

Cheng, T.Y., Chen, M.H., Chang, W.H., Huang, M.Y., and Wang, T.W. (2013). Neural stem cells encapsulated in a functionalized self-assembling peptide hydrogel for brain tissue engineering. Biomaterials 34, 2005-2016.

Chivatakarn, O., Kaneko, S., He, Z., Tessier-Lavigne, M., and Giger, R.J. (2007). The Nogo-66 receptor NgR1 is required only for the acute growth cone-collapsing but not the chronic growth-inhibitory actions of myelin inhibitors. J Neurosci 27, 7117-7124.

Chung, K.Y., Taylor, J.S.H., Shum, D.K.Y., and Chan, S.O. (2000). Axon routing at the optic chiasm after enzymatic removal of chondroitin sulfate in mouse embryos. Development 127, 2673-2683.

Cragg, B. (1979). Brain extracellular space fixed for electron microscopy. Neurosci Lett 15, 301-306.

Cunha, C., Panseri, S., Villa, O., Silva, D., and Gelain, F. (2011). 3D culture of adult mouse neural stem cells within functionalized self-assembling peptide scaffolds. Int J Nanomedicine 6, 943-955.

Dalby, M.J., Riehle, M.O., Johnstone, H., Affrossman, S., and Curtis, A.S. (2002a). In vitro reaction of endothelial cells to polymer demixed nanotopography. Biomaterials 23, 2945-2954.

Dalby, M.J., Riehle, M.O., Johnstone, H.J., Affrossman, S., and Curtis, A.S. (2002b). Polymer-demixed nanotopography: control of fibroblast spreading and proliferation. Tissue Eng 8, 1099-1108. 
Dalby, M.J., Yarwood, S.J., Riehle, M.O., Johnstone, H.J.H., Affrossman, S., and Curtsi, A.S.G. (2002c). Increasing fibroblast response to materials using nanotopography: morphological and genetic measurements of cell response to 13-nm-high polymer demixed islands. Exp Cell Res 276, 1-9.

Dityatev, A. and Schachner, M. (2003). Extracellular matrix molecules and synaptic plasticity. Nat Rev Neurosci 4, 456-468.

Domeniconi, M., Cao, Z.U., Spencer, T., Sivasankaran, R., Wang, K.C., Nikulina, E., Kimura, N., Cai, H., Deng, K.W., Gao, Y., et al. (2002). Myelin-associated glycoprotein interacts with the Nogo66 receptor to inhibit neurite outgrowth. Neuron 35, 283-290.

Engler, A.J., Sen, S., Sweeney, H.L., and Discher, D.E. (2006). Matrix elasticity directs stem cell lineage specification. Cell 126, 677-689.

Faulkner, J.R., Herrmann, J.E., Woo, M.J., Tansey, K.E., Doan, N.B., and Sofroniew, M.V. (2004). Reactive astrocytes protect tissue and preserve function after spinal cord injury. J Neurosci 24, 2143-2155.

Faux, C., Hawadle, M., Nixon, J., Wallace, A., Lee, S., Murray, S., and Stoker, A. (2007). PTPsigma binds and dephosphorylates neurotrophin receptors and can suppress NGF-dependent neurite outgrowth from sensory neurons. Biochim Biophys Acta 1773, 1689-1700.

Fawcett, J.W. and Keynes, R.J. (1990). Peripheral nerve regeneration. Annu Rev Neurosci 13, 43-60.

Ferrari, A., Cecchini, M., Dhawan, A., Micera, S., Tonazzini, I., Stabile, R., Pisignano, D., and Beltram, F. (2011). Nanotopographic control of neuronal polarity. Nano Lett 11, 505-511.

Flanagan, L.A., Ju, Y.E., Marg, B., Osterfield, M., and Janmey, P.A. (2002). Neurite branching on deformable substrates. Neuroreport 13, 2411-2415.

Fournier, A.E., GrandPre, T., and Strittmatter, S.M. (2001). Identification of a receptor mediating Nogo-66 inhibition of axonal regeneration. Nature 409, 341-346.

Gallagher, J.O., McGhee, K.F., Wilkinson, C.D., and Riehle, M.O. (2002). Interaction of animal cells with ordered nanotopography. IEEE Trans Nanobiosc 1, 24-28.

Gao, Y., Deng, K.W., Hou, J.W., Bryson, J.B., Barco, A., Nikulina, E., Spencer, T., Mellado, W., Kandel, E.R., and Filbin, M.T. (2004). Activated CREB is sufficient to overcome inhibitors in myelin and promote spinal axon regeneration in vivo. Neuron 44, 609-621.

Gaudet, A.D., Popovich, P.G., and Ramer, M.S. (2011). Wallerian degeneration: gaining perspective on inflammatory events after peripheral nerve injury. J Neuroinflammation 8, 110 .

Georges, P.C., Miller, W.J., Meaney, D.F., Sawyer, E.S., and Janmey, P.A. (2006). Matrices with compliance comparable to that of brain tissue select neuronal over glial growth in mixed cortical cultures. Biophys J 90, 3012-3018. 
Giger, R.J., Hollis, E.R., and Tuszynski, M.H. (2010). Guidance molecules in axon regeneration. Cold Spring Harb Perspect Biol 2, a001867.

Gunn, J.W., Turner, S.D., and Mann, B.K. (2005). Adhesive and mechanical properties of hydrogels influence neurite extension. J Biomed Mater Res A 72A, 91-97.

Halliday, N.L. and Tomasek, J.J. (1995). Mechanical properties of the extracellular matrix influence fibronectin fibril assembly in vitro. Exp Cell Res 217, $109-117$.

Harrison, B.S. and Atala, A. (2007). Carbon nanotube applications for tissue engineering. Biomaterials 28, 344-353.

Her, G.J., Wu, H.C., Chen, M.H., Chen, M.Y., Chang, S.C., and Wang, T.W. (2013). Control of three-dimensional substrate stiffness to manipulate mesenchymal stem cell fate toward neuronal or glial lineages. Acta Biomater 9, 5170-5180.

Hodgkin, A.L. and Huxley, A.F. (1952). A quantitative description of membrane current and its application to conduction and excitation in nerve. J Physiol 117, $500-544$.

Hu, H., Ni, Y., Montana, V., Haddon, R.C., and Parpura, V. (2004). Chemically functionalized carbon nanotubes as substrates for neuronal growth. Nano Lett 4, 507-511.

Huang, Y.J., Wu, H.C., Tai, N.H., and Wang, T.W. (2012). Carbon nanotube rope with electrical stimulation promotes the differentiation and maturity of neural stem cells. Small 8, 2869-2877.

Jiang, F.X., Yurke, B., Firestein, B.L., and Langrana, N.A. (2008). Neurite outgrowth on a DNA crosslinked hydrogel with tunable stiffnesses. Ann Biomed Eng 36, 1565-1579.

Kabiri, M., Soleimani, M., Shabani, I., Futrega, K., Ghaemi, N., Ahvaz, H.H., Elahi, E., and Doran, M.R. (2012). Neural differentiation of mouse embryonic stem cells on conductive nanofiber scaffolds. Biotechnol Lett 34, 1357-1365.

Kantor, D.B., Chivatakarn, O., Peer, K.L., Oster, S.F., Inatani, M., Hansen, M.J., Flanagan, J.G., Yamaguchi, Y., Sretavan, D.W., Giger, R.J., et al. (2004). Semaphorin $5 \mathrm{~A}$ is a bifunctional axon guidance cue regulated by heparan and chondroitin sulfate proteoglycans. Neuron 44, 961-975.

Kim, J.E., Liu, B.P., Park, J.H., and Strittmatter, S.M. (2004). Nogo-66 receptor prevents raphespinal and rubrospinal axon regeneration and limits functional recovery from spinal cord injury. Neuron 44, 439-451.

Koh, H.S., Yong, T., Chan, C.K., and Ramakrishna, S. (2008). Enhancement of neurite outgrowth using nano-structured scaffolds coupled with laminin. Biomaterials 29, 3574-3582.

Kotov, N.A., Winter, J.O., Clements, I.P., Jan, E., Timko, B.P., Campidelli, S., Pathak, S., Mazzatenta, A., Lieber, C.M., Prato, M., et al. (2009). Nanomaterials for neural interfaces. Adv Mater 21, 3970-4004. 
Kubinova, S., Horak, D., Kozubenko, N., Vanecek, V., Proks, V., Price, J., Cocks, G., and Sykova, E. (2010). The use of superporous Ac-CGGASIKVAVS-OHmodified PHEMA scaffolds to promote cell adhesion and the differentiation of human fetal neural precursors. Biomaterials 31, 5966-5975.

Kubota, Y., Morita, T., Kusakabe, M., Sakakura, T., and Ito, K. (1999). Spatial and temporal changes in chondroitin sulfate distribution in the sclerotome play an essential role in the formation of migration patterns of mouse neural crest cells. Dev Dyn 214, 55-65.

Kwok, J.C., Carulli, D., and Fawcett, J.W. (2010). In vitro modeling of perineuronal nets: hyaluronan synthase and link protein are necessary for their formation and integrity. J Neurochem 114, 1447-1459.

Lamour, G., Journiac, N., Souès, S., Bonneau, S., Nassoy, P., Hamraoui, A. (2009). Influence of surface energy distribution on neuritogenesis. Colloids Surf B Biointerfaces 72(2), 208-218.

Lamour, G., Eftekhari-Bafrooei, A., Borguet, E., Soues, S., and Hamraoui, A. (2010). Neuronal adhesion and differentiation driven by nanoscale surface free-energy gradients. Biomaterials 31, 3762-3771.

Lau, L.W., Cua, R., Keough, M.B., Haylock-Jacobs, S., and Yong, V.W. (2013). Pathophysiology of the brain extracellular matrix: a new target for remyelination. Nat Rev Neurosci 14, 722-729.

Leach, J.B., Brown, X.Q., Jacot, J.G., DiMilla, P.A., and Wong, J.Y. (2007). Neurite outgrowth and branching of PC12 cells on very soft substrates sharply decreases below a threshold of substrate rigidity. J Neural Eng 4, 26-34.

Lee, M.R., Kwon, K.W., Jung, H., Kim, H.N., Suh, K.Y., Kim, K., and Kim, K.S. (2010). Direct differentiation of human embryonic stem cells into selective neurons on nanoscale ridge/groove pattern arrays. Biomaterials 31 , 4360-4366.

Leipzig, N.D. and Shoichet, M.S. (2009). The effect of substrate stiffness on adult neural stem cell behavior. Biomaterials 30, 6867-6878.

Liu, B.P., Fournier, A., GrandPre, T., and Strittmatter, S.M. (2002). Myelinassociated glycoprotein as a functional ligand for the Nogo-66 receptor. Science 297, 1190-1193.

Liu, K., Tedeschi, A., Park, K.K., and He, Z.G. (2011). Neuronal intrinsic mechanisms of axon regeneration. Annu Rev Neurosci, 34, 131-152.

Ma, W., Tavakoli, T., Derby, E., Serebryakova, Y., Rao, M.S., and Mattson, M.P. (2008). Cell-extracellular matrix interactions regulate neural differentiation of human embryonic stem cells. BMC Dev Biol 8, 90.

Maeda, N. and Noda, M. (1996). 6B4 proteoglycan/phosphacan is a repulsive substratum but promotes morphological differentiation of cortical neurons. Development 122, 647-658. 
Malarkey, E.B., Reyes, R.C., Zhao, B., Haddon, R.C., and Parpura, V. (2008). Water soluble single-walled carbon nanotubes inhibit stimulated endocytosis in neurons. Nano Lett 8, 3538-3542.

Mammadov, B., Mammadov, R., Guler, M.O., and Tekinay, A.B. (2012a). Cooperative effect of heparan sulfate and laminin mimetic peptide nanofibers on the promotion of neurite outgrowth. Acta Biomater 8, 2077-2086.

Mammadov, R., Mammadov, B., Guler, M.O., and Tekinay, A.B. (2012b). Growth factor binding on heparin mimetic peptide nanofibers. Biomacromolecules 13, 3311-3319.

Margolis, R.K., Rauch, U., Maurel, P., and Margolis, R.U. (1996). Neurocan and phosphacan: two major nervous tissue-specific chondroitin sulfate proteoglycans. Perspect Dev Neurobiol 3, 273-290.

Martini, R., Fischer, S., Lopez-Vales, R., and David, S. (2008). Interactions between Schwann cells and macrophages in injury and inherited demyelinating disease. Glia 56, 1566-1577.

Mckeon, R.J., Hoke, A., and Silver, J. (1995). Injury-induced proteoglycans inhibit the potential for laminin-mediated axon growth on astrocytic scars. Exp Neurol 136, 32-43.

Meyer-Franke, A., Wilkinson, G.A., Kruttgen, A., Hu, M., Munro, E., Hanson, M.G., Reichardt, L.F., and Barres, B.A. (1998). Depolarization and cAMP elevation rapidly recruit $\operatorname{TrkB}$ to the plasma membrane of CNS neurons. Neuron 21, 681-693.

Migliorini, E., Grenci, G., Ban, J., Pozzato, A., Tormen, M., Lazzarino, M., Torre, V., and Ruaro, M.E. (2011). Acceleration of neuronal precursors differentiation induced by substrate nanotopography. Biotechnol Bioeng 108, 2736-2746.

Moreau-Fauvarque, C., Kumanogoh, A., Camand, E., Jaillard, C., Barbin, G., Boquet, I., Love, C., Jones, E.Y., Kikutani, H., Lubetzki, C., et al. (2003). The transmembrane semaphorin Sema4D/CD100, an inhibitor of axonal growth, is expressed on oligodendrocytes and upregulated after CNS lesion. J Neurosci 23, 9229-9239.

Murnane, A.C., Brown, K., and Keith, C.H. (2002). Preferential initiation of PC12 neurites in directions of changing substrate adhesivity. J Neurosci Res 67(3), $321-328$.

Nakajima, M., Ishimuro, T., Kato, K., Ko, I.K., Hirata, I., Arima, Y., and Iwata, H. (2007). Combinatorial protein display for the cell-based screening of biomaterials that direct neural stem cell differentiation. Biomaterials 28, 1048-1060.

Neumann, S., Bradke, F., Tessier-Lavigne, M., and Basbaum, A.I. (2002). Regeneration of sensory axons within the injured spinal cord induced by intraganglionic cAMP elevation. Neuron 34, 885-893.

Niederost, B.P., Zimmermann, D.R., Schwab, M.E., and Bandtlow, C.E. (1999). Bovine CNS myelin contains neurite growth-inhibitory activity associated with chondroitin sulfate proteoglycans. J Neurosci 19, 8979-8989. 
Pardo, B. and Honegger, P. (2000). Differentiation of rat striatal embryonic stem cells in vitro: monolayer culture vs. three-dimensional coculture with differentiated brain cells. J Neurosci Res 59, 504-512.

Pasterkamp, R.J. and Verhaagen, J. (2006). Semaphorins in axon regeneration: developmental guidance molecules gone wrong? Philos Trans R Soc Lond B Biol Sci 361, 1499-1511.

Peretz, H., Talpalar, A.E., Vago, R., and Baranes, D. (2007). Superior survival and durability of neurons and astrocytes on 3-dimensional aragonite biomatrices. Tissue Eng 13, 461-472.

Prabhakaran, M.P., Venugopal, J.R., and Ramakrishna, S. (2009). Mesenchymal stem cell differentiation to neuronal cells on electrospun nanofibrous substrates for nerve tissue engineering. Biomaterials 30, 4996-5003.

Qiu, J., Cai, C.M., Dai, H.N., McAtee, M., Hoffman, P.N., Bregman, B.S., and Filbin, M.T. (2002). Spinal axon regeneration induced by elevation of cyclic AMP. Neuron 34, 895-903.

Ren, Y.J., Zhang, H., Huang, H., Wang, X.M., Zhou, Z.Y., Cui, F.Z., and An, Y.H. (2009). In vitro behavior of neural stem cells in response to different chemical functional groups. Biomaterials 30, 1036-1044.

Riopelle, R.J. and Dow, K.E. (1990). Functional interactions of neuronal heparansulfate proteoglycans with laminin. Brain Res 525, 92-100.

Sabri, F., Cole, J.A., Scarbrough, M.C., and Leventis, N. (2012). Investigation of polyurea-crosslinked silica aerogels as a neuronal scaffold: a pilot study. PLoS One 7, e33242.

Saha, K., Keung, A.J., Irwin, E.F., Li, Y., Little, L., Schaffer, D.V., and Healy, K.E. (2008). Substrate modulus directs neural stem cell behavior. Biophys J 95, 4426-4438.

Schmalfeldt, M., Bandtlow, C.E., Dours-Zimmermann, M.T., Winterhalter, K.H., and Zimmermann, D.R. (2000). Brain derived versican V2 is a potent inhibitor of axonal growth. J Cell Sci 113, 807-816.

Sharma, K., Selzer, M.E., and Li, S. (2012). Scar-mediated inhibition and CSPG receptors in the CNS. Exp Neurol 237, 370-378.

Silva, G.A., Czeisler, C., Niece, K.L., Beniash, E., Harrington, D.A., Kessler, J.A., and Stupp, S.I. (2004). Selective differentiation of neural progenitor cells by high-epitope density nanofibers. Science 303, 1352-1355.

Snider, W.D., Zhou, F.Q., Zhong, J., and Markus, A. (2002). Signaling the pathway to regeneration. Neuron 35, 13-16.

Snow, D.M., Lemmon, V., Carrino, D.A., Caplan, A.I., and Silver, J. (1990). Sulfated proteoglycans in astroglial barriers inhibit neurite outgrowth in vitro. Exp Neurol 109, 111-130.

Stabenfeldt, S.E., Munglani, G., Garcia, A.J., and LaPlaca, M.C. (2010). Biomimetic microenvironment modulates neural stem cell survival, migration, and differentiation. Tissue Eng Part A 16, 3747-3758. 
Sur, S., Pashuck, E.T., Guler, M.O., Ito, M., Stupp, S.I., and Launey, T. (2012). A hybrid nanofiber matrix to control the survival and maturation of brain neurons. Biomaterials 33, 545-555.

Tani, E. and Ametani, T. (1971). Extracellular distribution of ruthenium redpositive substance in the cerebral cortex. J Ultrastruct Res 34, 1-14.

Teixeira, A.I., Ilkhanizadeh, S., Wigenius, J.A., Duckworth, J.K., Inganas, O., and Hermanson, O. (2009). The promotion of neuronal maturation on soft substrates. Biomaterials 30, 4567-4572.

Tu, Q., Li, L., Zhang, Y.R., Wang, J.C., Liu, R., Li, M.L., Liu, W.M., Wang, X.Q., Ren, L., and Wang, J.Y. (2011). The effect of acetylcholine-like biomimetic polymers on neuronal growth. Biomaterials 32, 3253-3264.

Walicke, P.A. (1988). Interactions between basic fibroblast growth factor (FGF) and glycosoaminoglycans in promoting neurite outgrowth. Exp Neurol 102, $144-148$.

Wang, K.C., Koprivica, V., Kim, J.A., Sivasankaran, R., Guo, Y., Neve, R.L., and He, Z.G. (2002). Oligodendrocyte-myelin glycoprotein is a Nogo receptor ligand that inhibits neurite outgrowth. Nature 417, 941-944.

Willits, R.K. and Skornia, S.L. (2004). Effect of collagen gel stiffness on neurite extension. J Biomater Sci Polym Ed 15, 1521-1531.

Winzeler, A.M., Mandemakers, W.J., Sun, M.Z., Stafford, M., Phillips, C.T., and Barres, B.A. (2011). The lipid sulfatide is a novel myelin-associated inhibitor of CNS axon outgrowth. J Neurosci 31, 6481-6492.

Yamada, H., Fredette, B., Shitara, K., Hagihara, K., Miura, R., Ranscht, B., Stallcup, W.B., and Yamaguchi, Y. (1997). The brain chondroitin sulfate proteoglycan brevican associates with astrocytes ensheathing cerebellar glomeruli and inhibits neurite outgrowth from granule neurons. J Neurosci 17, 7784-7795.

Yamaguchi, Y. (2000). Lecticans: organizers of the brain extracellular matrix. Cell Mol Life Sci 57, 276-289.

Yiu, G. and He, Z.G. (2006). Glial inhibition of CNS axon regeneration. Nat Rev Neurosci 7, 617-627.

Yu, T.T. and Shoichet, M.S. (2005). Guided cell adhesion and outgrowth in peptide-modified channels for neural tissue engineering. Biomaterials 26, 1507-1514. 